Aus der I.Medizinischen Klinik (Dir.: Prof.Dr.H.Krosch) der Martin-Luther-Universität Halle-Wittenberg

\title{
Franz Leopold de Lafontaine (1756-1812) aus Rorschach, ein Schweizer Wundarzt als hallescher Doktorand des Jahres 1791
}

\section{Von Wolfram Kaiser}

Noch während des gesamten 18. Jahrhunderts liegt in Deutschland die praktische Chirurgie vorwiegend in den Händen der zunftmäßig organisierten Chirurgen, Bader und Barbiere ${ }^{7,8}$; an den Universitäten wird eine «Chirurgia medica» gelesen, welche sich ausschließlich auf diejenigen Grenzgebiete bezieht, wo sich konventionelle Medizin und handwerkliche Chirurgie überschneiden. Nur allmählich löst sich letztere aus der Zunftgebundenheit und unterstellt sich den lokalen Medizinalbehörden.

Derartige Regelungen werden in den einzelnen Ländern des damaligen Deutschen Reiches recht unterschiedlich gehandhabt; an der grundsätzlichen Einstellung ändert sich aber kaum etwas - ganz im Gegensatz z.B. zu Frankreich, wo die Ars chirurgica durch die Leistungen hervorragender Wundärzte zu hoher Blüte und großem Ansehen gelangt.

Die Medizinische Fakultät Paris fragt im Januar 1749 bei der Universität Halle an, wie man es hierzulande mit der Rechtsstellung von Ärzten und Chirurgen halte; im Fakultätstagebuch der Academia Fridericiana ist diese «responsio ad litteras Facultatis Medicae Parisiensis, de ordine, officiis et relatione Chirurgorum ad Medicos, horumque praerogativa» für den 23. Januar 1749 eingetragen ${ }^{16}$. Sieben Jahre später präzisiert der amtierende hallesche Dekan Andreas Elias Büchner (1701-1769) den in der Saalestadt vertretenen Standpunkt, als er am 6. April 1756 an gleicher Stelle notiert ${ }^{17}$ : «ist ein Responsum vor die Barbierer und Wundärtzte in Nürnberg ertheilet worden, daß sie von denen gemeinen Handwercks-Zünften abzusondern, und als Angehörige von dem dasigen Collegio medico zu halten sind».

Der Chirurg rangiert im Preußen des 18. Jahrhunderts nach den «Medicinae Doctores» und den «Medicinae Practici» an dritter Stelle der medizinischen Rangordnung. Allerdings verschließt man sich an den Landesuniversitäten keineswegs vor den im Ausland erzielten Fortschritten in dieser Fachrichtung. Wenn der Absolvent einer Chirurgenschule bzw. ein schon in der Praxis tätig gewesener Wundarzt sich nachträglich um das akademische Medizinstudium bemüht, wird ihm hierauf eine gewisse Zeit angerechnet, d.h. er darf bei guten Leistungen mit einer verkürzten Studien- 
dauer rechnen. In seltenen Einzelfällen ist man sogar bereit, auf Grund besonderer wissenschaftlicher Vorleistungen verdiente Chirurgen formell zum Dr.med. zu promovieren ${ }^{6}$. Dieser Fall ist an der Academia Fridericiana Halensis insgesamt achtmal praktiziert worden, u.a. am 6. August 1762 für den berühmten Antoine Louis (1723-1792), Schüler von Francois Gigot de la Peyronie (1678-1747) und Mitglied der Französischen Akademie der Wissenschaften. Der Eintragung im halleschen Fakultätstagebuch ist zu entnehmen, daß diese Ehrung «speciatim a Facultate nostra» erbeten war ${ }^{14}$.

Unter diesen acht in Anerkennung ihrer Verdienste mit dem Doktortitel geehrten Chirurgen befinden sich drei Schweizer Wundärzte. Es sind die Militärchirurgen Johann Ulrich Bilguer aus Chur (am 21.März 1761) und Johann Heinrich Roth aus Keßwil (am 26.Oktober 1761) sowie der in polnischen Diensten stehende Wundarzt Franz Leopold de Lafontaine aus Rorschach (am 22.November 1791). Die Beziehungen des berühmten Johann Ulrich Bilguer zur Medizinischen Fakultät Halle wurden vor kurzem ausführlich abgehandelt ${ }^{3}$; die 30 Jahre nach der Bilguerschen Promotion erfolgende Coronatio doctoralis von Franz Leopold de Lafontaine (1756-1812) ist dagegen wenig bekannt - ganz abgesehen davon, daß er in der medizinisch-biographischen Literatur 1, 2,9 als «Schwabe» geführt wird.

Franz Leopold de Lafontaine ist nach eigenen Angaben ${ }^{18}$ am 14. Januar 1756 in Rorschach in der Schweiz geboren. Im Anschluß an die Grundausbildung, über die er in seinem Curriculum vitae keine näheren Angaben macht, erlernt er in einer Offizin für vier Jahre die Grundlagen der Ars pharmaceutica. 1775 begibt er sich als Medizinstudent nach Straßburg; hier hört er u. a. bei Johann Reinhold Spielmann (1722-1783), Johann Friedrich Lobstein (1736-1784) und Johann Christian Ehrmann (1749-1827). 1775 erwirbt Lafontaine den Grad eines Magister Chirurgiae: dann geht er bis 1781 nach Wien und im Anschluß als praktischer Wundarzt nach Krakau. Von hier aus beruft ihn Stanislaw Poniatowski als Protochirurg mit dem Titel eines Hofrats nach Warschau.

Im Jahre 1791 nimmt Franz Leopold de Lafontaine Kontakt zu den Medizinischen Fakultäten von Halle und Straßburg auf, um die einst versäumte Promotion nachzuholen. Sein russischer Kollege Heinrich von Kelchen ${ }^{12}$ war 1790 von Marburg aus in absentia promoviert worden - das bestärkt Lafontaine offenbar zu einem gleichartigen Ansuchen, welches er im Sommer 1791 schriftlich einleitet.

Lafontaine hatte bis dahin bereits eine Reihe von Arbeiten veröffentlicht, die er bei seiner Antragstellung aufführt, um seine wissenschaftliche Quali- 
fikation nachzuweisen. Während der Krakauer Tätigkeit hatte er sich u.a. um die Neueinrichtung des Bades Krzeszowice bemüht und 1789 eine balneologische Schrift in polnischer Sprache herausgebracht. Als er sich an die Academia Fridericiana nach Halle wendet, sind außerdem bereits die «Chirurgisch-medicinischen Wahrnehmungen Polen betreffend» im Druck (Leipzig 1792).

Am 29. August 1791 schreibt Lafontaine an den halleschen Privatdozenten Dr.Gottlieb Karl Jacob (1764-1813) und bittet ihn um Vermittlung. Letzterer steht in Korrespondenz mit dem polnischen Chirurgen Michael Wolfgang v. Kortowsky ${ }^{5}$ - über ihn dürfte die Verbindung hergestellt worden sein. Lafontaine weist dabei darauf hin, er habe in Wien und Krakau Privatkollegs gelesen und benötige jetzt den Doktortitel, weil «in Polen die Chirurgen so verachtet sind ».

Der Antrag erfolgt zu einem Zeitpunkt, als sich in Polen politische und wirtschaftliche Entscheidungen von weittragender Bedeutung anbahnen. Das Land hatte nach der ersten Teilung einen wirtschaftlichen Aufschwung erlebt ${ }^{10,13}$; Reformversuche patriotischer Kräfte hatten zu einer am 3.Mai 1791 angenommenen Verfassung geführt, gleichzeitig aber neue Wirren ausgelöst, die zur zweiten Teilung führen sollten.

Der Lafontainesche Antrag liegt der halleschen Fakultät am 24.Oktober 1791 vor. Diese äußert sich zustimmend, verlangt aber die Nachreichung eines Curriculum vitae. Dieses trifft drei Wochen später ein und lautet ${ }^{18}$ :

«Franciscus Leopoldus de Lafontaine ... qui natus in oppido Helvetiorum Rorschach anno 1756. d.14. Januarii, parentibus Leopoldo de Lafontaine et Francisca ex gente Leonhardorum, iisque artibus, quibus aetas juvenilis impartiti solet, eruditus, cum satis jam necessariis subsidiis instructus viderer, primis adolescentiae annis in officina medicamentorum per quadriennium ediscendae arti pharmaceuticae operam navavi. Dein, cum ad altiora animus fertet, anno huius seculi septingentesimo quinto Argentoratum me contuli, ibidemque per quator annos ducibus celeberrimis ad aeternum colendis praeceptoribus Lobstein, Pfeffinger, Spielmann, Ehrmann, Isengarth, Diebold, Guering, praelectionibus Physicae, Anatomiae, Physiologiae, Botanices, Materiae medicae, Chemiae, Pathologiae tam generalis quam specialis, nec non laboribus clinicis et operationibus chirurgicis assiduum me praebui. Quibus absolutis, cum per triduum examen rigorosum habuissem, exhibito etiam in Theatro anatomico operationum chirurgicarum Specimine, Magistri Chirurgiae honoribus auctus sum. Peractis Argentorati studiis, peragratisque discendi consilio finitissimis terris, anno hujus seculi septingentesimo nono Viennam profectus sum, ibidemque per biennium non neglectis plurimorum in arte medica insignium virorum praelectionibus, praecipue tamen in cognoscendis et curandis ad lectos aegrotantium morbis usus sum duce et auspice heu! nimis mature erepto nobis Celeberrimo Stoll.

Elapso biennio isto Cracoviam me contuli, ubi cum aliquantopere artem medicam exercuissem, quatuor abhinc annis, a Serenissimo et Potentissimo Poloniae Rege ad abeundum Protochirurgi munus gratiosissime accessitus, Varsaviam perveni. 
Vestrum jam erit, Spectatissimi, Fautores ac Domini summa pietate colendi, judicare, utrum dignus Vobis esse videar, cui summos in Medicina et Chirurgia honores conferatis, quibus ut me quam gratiosissime augere et ornare velitis, ea, qua par est, observantia oro rogoque: dissertationem inauguralem de plica polonica, quam, variis muneris occupationibus districtus, perpolire adeo ut in lucem prodire possit, impraesentiarum prohibeor, propediem Vestro, Viri Clarissimi, examini subiecturus.»

Die hallesche Fakultät glaubt nunmehr, die Fertigstellung der vorgesehenen Arbeit «de plica polonica» nicht abwarten zu müssen, um eine Doktorpromotion für Lafontaine aussprechen zu dürfen. Am 22. November 1791 teilt der Dekan Kemme den Fakultätsmitgliedern mit, er habe das Curriculum vitae, die Promotionsgebühren und «den untersiegelten Eyd» des Antragstellers erhalten; ein Diplomentwurf für Lafontaine geht ebenfalls in den Umlauf. Erneut stimmen die halleschen Professoren zu; lediglich Johann Christian Reil (1759-1813) macht auf eine Passage im Diplomvordruck aufmerksam, die vielleicht ein Versehen, möglicherweise aber auch ein bewußtes Politicum darstellt. Es heißt dort nämlich bei den aufgeführten Titeln des Doktoraspiranten: «Chirurgiae Magistro, Serenissimo ac Potentissimo Poloniarum Regi a Consiliis Aulae.» Reil fragt: «Muß es im Diplom nicht entweder Poloniae Regis oder Polonorum Regis heißen?» Die aufgeführte - und später im Originaldiplom auch nicht korrigierte - Passage bedeutet nämlich «König beider Polen» bzw. sachlich « König von Gesamtpolen »: das weist auf den Rechtsanspruch eines Landes hin, welches durch erzwungene Abtretungen auf ein Restgebiet beschränkt war und nach dem erfolglosen Aufstand von Kosciuszko (1794) dann eine dritte Aufteilung unter die Großmächte Rußland, Österreich und Preußen über sich ergehen lassen muß. Vielleicht hat hier ein Setzer bzw. ein Angehöriger der halleschen Druckerei seiner Einstellung Ausdruck gegeben, als er «Rex Poloniarum » notierte.

Der «untersiegelte Eyd» von Lafontaine hat folgenden Wortlaut: «Ego Franciscus Leopoldus de Lafontaine, Serenissimo ac Potentissimo Poloniae Regi a consiliis aulae, Eiusdemque S.R.M. Protochirurgus, juro, me Serenissimo atque Potentissimo Regi Borussiae fidelem futurum, commoda Regiae Majestatis, Eiusque Domus, ut et Academiae hujus pro viribus promoturum, Statutis Regiis, et Facultati Medicae reverentiam habiturum, et si ad ipsos aegros sanandos requisitus fuerim, nihil dolose sed omnia circumspecte secundum conscientiam et regulas medicas acturum, expediturumque, et non tam meum commodum, quam aegri sanitatem et salutem, sive pauper sive dives sit, quaesiturum.»

Lafontaine ist nunmehr Doctor Medicinae et Chirurgiae ${ }^{15}$; einige andere polnische Chirurgen folgen seinem Beispiel und werden von Halle aus ebenfalls promoviert ${ }^{5}$. Lafontaine bleibt auch dann in Polen, als das Land unter 
den Okkupanten aufgeteilt wird. In Warschau gibt er 1801 und 1802 die Monatsschrift Dziennik zdrowia dla wszystkich stanow (Gesundheitszeitung für alle Stände) heraus, in welcher er in Anlehnung an Ramazzini und Johann Christian Gottlieb Ackermann (1756-1801) berufsmedizinische Probleme aufgreift ${ }^{13}$. Aus diesen Abhandlungen übersetzt 1808 Antoine-Jaques-Louis Jourdan (1788-1848) das Kapitel über den Weichselzopf ins Französische. An Kampfhandlungen während der polnischen Aufstandsbewegungen scheint Lafontaine nicht beteiligt gewesen zu sein - zumindest wird sein Name in den sonst sehr ausführlichen Berichten des preußisch-deutschen Militärarztes Ludwig Formey nicht genannt ${ }^{11}$. Als dann aber Napoleon das Großherzogtum Warschau begründet, beruft Jozef Poniatowski, der Oberbefehlshaber der polnischen Armee, Lafontaine im Jahre 1807 zum Chef des Heeressanitätsdienstes und zum General-Protochirurgen. Im russischen Feldzug von 1812 gerät Lafontaine bei Moskau in Gefangenschaft und stirbt im Dezember des gleichen Jahres in Mohilew. Sein hallescher «Moderator» Jacob wird 1813 ebenfalls ein Opfer der Napoleonischen Kriege ${ }^{4}$.

Es ist nichts darüber bekannt, ob Franz Leopold de Lafontaine nach der Promotion von 1791 noch Beziehungen nach Halle unterhalten hat; auch scheint er seine Schweizer Heimat nicht wieder besucht zu haben. Seine hinterlassenen Schriften wurden 1824 durch Jeremias Rudolph Lichtenstaedt (1792-1849) unter dem Namen des Verfassers herausgegeben. Die Besonderheiten des Promotionsverfahrens und die bemerkenswerten Leistungen dieses Schweizer Chirurgen in polnischen Diensten gaben Anlaß zu der vorliegenden Studie. 


\section{Schrifttum}

1. Hirsch A. und E. Gurlt, Biographisches Lexikon der hervorragenden Ärzte aller Zeiten und Völker, Band 3, 1.Auflage, Wien/Leipzig 1886

2. Dieselben, Biographisches Lexikon der herorragenden Ärzte aller Zeiten und Völker, Band 3, 2.Auflage, bearbeitet von W.Haberling, F.Hüвotter und H.Vierordt, Berlin/Wien 1931

3. KaISER W., Johann Ulrich Bilguer (1720-1796) und die Medizinische Fakultät Halle, Gesnerus 27 (1970) 85

4. KaISer W. und H. Krosch, Zur Geschichte der Medizinischen Fakultät der Universität Halle im 18. Jahrhundert (IX,X): Extraordinarien und Doctores legentes, Wiss. Z. Univ. Halle XIV (1965) 357

5. Dieselben, Wissenschaftsbeziehungen Halle-Rußland aus medizinhistorischer Sicht (18. Jahrhundert), Wiss. B. Univ. Halle (1967/4 R3). Halle 1967

6. Dieselben, Zur Anwendung von Studien- und Medizinaledikten des 18. Jahrhunderts an der Medizinischen Fakultät Halle, Beitr. Gesch. Univ. Erfurt 14 (1968) 139

7. Kaiser W., und W.Piechocki, Die Anfänge des chirurgischen Unterrichts an der Universität Halle, Dtsch. Ges.-Wes. 23 (1968) 1658

8. Dieselben, Zur Geschichte des halleschen Bader- und Chirurgenhandwerks, Med. Monatsschr.22 (1968) 399

9. Kosminski S., Slownik Lekarzow polskich, Warszawa 1888

10. Kula W. Ksztaltowanie sie kapitalizmu w Polsce, Warszawa 1955

11. Leibbrand W., Teilnahme eines Berliner Arztes am Polenfeldzug 1794, Dtsch. med. Wschr. 63 (1937) 1809

12. Richter W.M.v., Geschichte der Medicin in Rußland, Band 3, Moskau 1817

13. Schwann St., B.Ramazzinis «De morbis artificum diatriba» und die weitere Entwicklung der Berufspathologie im Osten Europas (Ungarn, Rußland, Polen), Wiss. Z. Univ. Berlin XVII (1968) 753

14. Univ.-Archiv Halle, Rep. 29 F VII, Nr. 1, pag. 49

15. Ebendort, Rep. 29 F VII, Nr.1, pag. 106

16. Ebendort, Rep. 29 F VII, Nr. 1, pag. 162

17. Ebendort, Rep. 29 F VII, Nr.1, pag. 168

18. Ebendort, Med. Fak. I, Nr. 27 